\title{
Volume change effect on the salt-finger stability of directionally solidifying ammonium chloride solution
}

\author{
Y.C. Lee, Falin Chen * \\ Institute of Applied Mechanics, National Taiwan University, Taipei, Taiwan, ROC
}

Received 26 December 1994; accepted 10 May 1995

\begin{abstract}
The effect of the volume change due to phase transformation on the stability of salt-finger convection of directionally solidifying $\mathrm{NH}_{4} \mathrm{Cl}$ aqueous solution cooling from below is investigated. The basic flow, induced by the volume change, not only changes the morphology and the depth of the dendritic mushy layer, but also influences the stability of salt-finger convection. A new mathematical model is proposed, which differs from the previous one mainly on the dynamical condition at the melt/mush interface. This difference not only leads to a less stable state, but can also be crucial to the dynamical behavior of the oscillatory instability mode since the convection cells of this mode are coupled viscously through the interface. In the discussion, special emphasis is placed on the volume change effect on the instability mode competition, which may be influential to the stability characteristics of the subsequent plume convection.
\end{abstract}

\section{Introduction}

Recently, a large research effort has been made to investigate the convective flows in the directional solidification of ammonium chloride solution cooling from below because of their significant influence on the composition as well as the structure of the resultant casting; especially the analogy between the flowinduced chimneys in the dendritic mushy zone and the freckles in the directionally solidified casting of metallic alloy, which causes a deleterious effect on the mechanical strength of the casting [1-10]. The details of the convective flows in solidifying $26 \% \mathrm{NH}_{4} \mathrm{Cl}$ solution are available in Chen and Chen [3]. Here, we briefly outline the principal flows in the following. At the beginning of solidification, due to the ejection of the lighter and cooler fluid from the solidification

\footnotetext{
${ }^{*}$ Corresponding author.
}

front, the salt-finger convection occurs. As the mushylayer grows to a certain height and, meanwhile, the salt-finger convection becomes more vigorous, convective plumes sparsely distributed on the plane of the solidification front come directly from the interior of the mush, leading to the formation of chimneys. Later on, the salt-finger convection fades out gradually while the plume convection becomes increasingly prevalent. The plume convection is believed to correspond to a subcritical mushy-layer mode instability [6] resulting from the perturbation of nonlinear salt-finger convection [10]. This scenario is supported by the experiment of Sample and Hellawell [1] that new plumes can be generated by withdrawing liquid in the vicinity above the freezing front. Accordingly, the stability of salt-finger convection has a close relation with the chimney formation.

Due to the importance of the salt-finger convection as stated above, several investigations have been de- 
voted to studying the stability characteristics of this flow. Tait \& Jaupart [2] investigated experimentally the onset of salt-finger convection by increasing the viscosity of $28 \% \mathrm{NH}_{4} \mathrm{Cl}$ solution and found that the onset of salt-finger convection occurs largely in the compositional boundary-layer above the melt/mush interface (the so-called boundary-layer mode convection) while the fluid in the mush does not participate in the initial instability. Worster [5] in a linear stability analysis investigated the stability of the mushy-layer mode convection, a convection circulating between the melt and the mush, for a special alloy. In another linear stability analysis Chen, Lu and Yang [10] concluded that the onset of salt-finger convection of $\mathrm{NH}_{4} \mathrm{Cl}$ solution is of boundary-layer mode and speculated that as the salt-finger convection becomes fully nonlinear the onset of the plume convection is induced. In another stability analysis Nandapurkar et al. [11] solved a set of dimensional equations particularly for the lead-tin alloy without considering the interaction between convection and solidification in the mushy layer. Their results, accordingly, are not relevant to the present study.

In the present paper, we examine the stability of salt-finger convection under the influence of volume change. The volume change, or the density change due to phase transformation, is significant in some alloys. As volume change occurs, either shrinking or expanding, a uniform flow is induced, which in turn influences the morphological instability of single component fluid [12]. For a binary solution, the induced flow not only changes the morphology of the dendritic mushy layer, but also varies the stability characteristics of the salt-finger convection. In the following paper, we present the mathematical formulation in Section 2, which is different from that of Worster [5] and Chen, Lu and Yang [10] in several respects. In Section 3 the basic state solution due to volume change is discussed. In Section 4 the linearized equations and method of solution are presented. In Section 5 the linear stability results are shown; special emphasis is placed on the convection modes and their stability criteria. In Section 6 , the conclusion is provided.

\section{Mathematical formulation}

Consider a mushy layer lying above a solid region and below a semi-infinite melt region. A binary solu- tion of concentration $C_{\infty}$ and temperature $T_{\infty}$ is directionally solidified from below and both the melt/mush and mush/solid interfaces move upwards with a constant velocity $V$. The mushy layer extends from $z=0$ to $z=h$, where $h$ is the position of the melt/mush interface which is to be determined as part of the solution. The temperature at the mush/solid interface is fixed at the eutectic temperature $T_{\mathrm{E}}$. Two sets of equations are required to describe the fluid motion of the system. Each set consists of the conservation of mass, momentum, heat, and solute, which are described in Fowler [13] and Chiareli and Worster [14] for the mushy layer and in McFadden and Coriell [15] for the fluid layer. The Boussinesq approximation is applied to the momentum equations of both layers. We nondimensionalize the fluid velocities with the prescribed interface velocity $V$, distances with $\kappa_{\mathrm{tl}} / V$, time with $\kappa_{\mathrm{tl}} / V^{2}$, density with $\rho_{\mathrm{l}}$, and pressure with $\beta \Delta C \rho_{1} g \kappa_{\mathrm{tl}} / V$. Here $\kappa_{\mathrm{t} l}$ is the thermal diffusivity of the fluid, $\beta=\beta^{*}-\Gamma \alpha^{*}, \beta^{*}$ and $\alpha^{*}$ are solutal and thermal expansion coefficients, respectively, $\Gamma$ is the slope of the liquidus curve, which is assumed to be a constant, $\Delta C=C_{\infty}-C_{\mathrm{E}}, C_{\mathrm{E}}$ is the eutectic concentration, $\rho_{1}$ is the density of the liquid, and $g$ is the gravitational acceleration. By assuming the physical properties, except the density, of the fluid and solid are identical and by taking the Galilean transformation with respect to the moving interface, we obtain the following dimensionless equations. In the fluid layer $h<z<\infty$, we have

$$
\begin{aligned}
& \nabla \cdot \boldsymbol{u}=0, \\
& {\left[\frac{\partial}{\partial t}-\frac{\partial}{\partial z}\right] \boldsymbol{\theta}+\boldsymbol{u} \cdot \nabla \theta=\nabla^{2} \theta,} \\
& {\left[\frac{\partial}{\partial t}-\frac{\partial}{\partial z}\right] \Theta+u \cdot \nabla \Theta=\epsilon \nabla^{2} \Theta,} \\
& \frac{1}{\sigma}\left[\left(\frac{\partial}{\partial t}-\frac{\partial}{\partial z}\right)+u \cdot \nabla\right] \boldsymbol{u} \\
& \quad=\nabla^{2} u+R_{\mathrm{T}} \theta e_{k}-R_{\mathrm{C}}\left(\Theta e_{k}+\frac{\beta}{\beta^{*}} \nabla p\right),
\end{aligned}
$$

where $u=(u, v, w)$ is the velocity vector in Cartesian coordinate. The dimensionless temperature and concentration are defined as

$\theta=\frac{T-T_{\mathrm{L}}\left(C_{\infty}\right)}{\Delta T}, \quad \Theta=\frac{C-C_{\infty}}{\Delta C}$, 
where $\Delta T=\Gamma \Delta C=T_{\mathrm{L}}\left(C_{\infty}\right)-T_{\mathrm{E}}, T_{\mathrm{L}}\left(C_{\infty}\right)$ is the liquidus temperature corresponding to $C_{\infty}, \epsilon=\kappa_{\mathrm{cl}} / \kappa_{\mathrm{tl}}$ is the Lewis number, $\kappa_{\mathrm{cl}}$ is the solutal diffusivity of the melt, $\sigma=\nu / \kappa_{\mathrm{tl}}$ is the Prandtl number, $e_{k}$ is the unit vector in the vertical direction, $p$ is the pressure, $R_{\mathrm{T}}$ and $R_{\mathrm{C}}$ are thermal and solutal Rayleigh numbers given by

$R_{\mathrm{T}}=\frac{g \alpha^{*} \Delta T H^{3}}{\kappa_{\mathrm{tl}} \nu}, \quad R_{\mathrm{C}}=\frac{g \beta^{*} \Delta C H^{3}}{\kappa_{\mathrm{tl}} \nu}$,

in which $\nu$ is the kinematic viscosity and $H=\kappa_{\mathrm{tl}} / V$ is the characteristic length. In the mushy-layer $0<z<$ $h$, we have [13]

$$
\begin{aligned}
& \left(\frac{\partial}{\partial t}-\frac{\partial}{\partial z}\right) \rho+\nabla \cdot(\rho \boldsymbol{u})=0 \\
& \left(\frac{\partial}{\partial t}-\frac{\partial}{\partial z}\right)[\rho(\Theta-\mathcal{C}) \chi]+\nabla \cdot[\rho \boldsymbol{u}(\Theta-\mathcal{C})] \\
& \quad=\epsilon \nabla \cdot(\chi \rho \nabla \Theta) \\
& \left(\frac{\partial}{\partial t}-\frac{\partial}{\partial z}\right) \theta+\boldsymbol{u} \cdot \nabla \theta \\
& \quad=\nabla^{2} \theta+\mathcal{F}\left(\frac{\partial}{\partial t}-\frac{\partial}{\partial z}\right)[\rho(1-\chi)] \\
& \frac{\boldsymbol{u}}{\Pi(\chi)}=-R_{\mathrm{m}}\left(\nabla p+\theta \boldsymbol{e}_{k}\right)
\end{aligned}
$$

where $\boldsymbol{u}$ is the barycentric velocity defined as $\boldsymbol{u}=\chi \boldsymbol{u}^{l}$ in which $\boldsymbol{u}^{l}$ is the liquid velocity in the mush, $\chi$ is the liquid mass fraction, and $\Pi$ is the dimensionless permeability of the mushy zone. Note that when volumechange vanishes, $\chi$ is also the liquid volume fraction (or porosity). In above equations, we also note that $\mathcal{C}=\left(C_{\mathrm{s}}-C_{\infty}\right) / \Delta C$ is the concentration ratio, $C_{\mathrm{s}}$ is the concentration of solid, $\mathcal{F}=\mathcal{L} / c \Delta T$ is the Stefan number, $\mathcal{L}$ is the latent heat of fusion, $c$ is the specific heat, and the Rayleigh number for the mushy layer is given by

$R_{\mathrm{m}}=\frac{g \beta \Delta C \Pi_{0} H}{\kappa_{\mathrm{t} 1} \nu}$

in which $\Pi_{0}$ is the reference permeability. Eqs. (7)(10) are derived according to Fowler's approach [13], which can also be obtained from the formulation of Chiareli and Worster [14] where they formulated the equations in terms of volume fraction and the Darcy velocity defined as $u_{D}=\rho \chi u^{1}$. In Eq. 9, we also assume the heat capacity per unit volume of both the liquid and solid phases to be the same. The liquidus relation $\theta=\Theta$ shall be applied in the mushy layer in which the thermodynamic equilibrium condition is assumed to be valid [6]. In Eqs. (7)-(9), the density is given as

$\rho=\frac{\rho_{\mathrm{s}}}{\rho_{1}} \frac{1}{1+\gamma \chi}$,

where $\gamma=\left(\rho_{\mathrm{s}}-\rho_{\mathrm{l}}\right) / \rho_{1}$ is a constant corresponding to the volume change and $\rho_{\mathrm{s}}$ and $\rho_{1}$ are densities of the solid and the liquid, respectively. If $\gamma<0$, the solid density is less than the melt density so that solidification results in an expansion as well as an upward flow, and vice versa for $\gamma>0$.

The boundary conditions at the $z \rightarrow \infty$ are

$$
\begin{aligned}
& \theta \rightarrow \theta_{\infty}, \\
& \Theta \rightarrow 0, \\
& w \rightarrow-\gamma .
\end{aligned}
$$

Eq. ( $13 \mathrm{c}$ ) corresponds to the flow induced by the volume change, see also Eq. (17) of Section 3. At the melt/mush interface $z=h$, the boundary conditions are

$$
\begin{aligned}
& \theta=\Theta, \\
& \boldsymbol{n} \cdot \nabla \theta=\boldsymbol{n} \cdot \nabla \Theta, \\
& {[\boldsymbol{n} \cdot \boldsymbol{u}]=0,} \\
& {[\theta]=0,} \\
& {[\boldsymbol{n} \cdot \nabla \theta]=0,} \\
& \chi=1, \\
& -\left.\frac{\beta}{\beta^{*}} R_{\mathrm{C}} p\right|_{h+}+\left.2 \frac{\partial w}{\partial z}\right|_{h+}=-\left.\mathcal{H} R_{\mathrm{m}} p\right|_{h-}, \\
& \left.\frac{\partial \boldsymbol{u}_{2}}{\partial z}\right|_{h+}=\frac{\Lambda \sqrt{\mathcal{H}}}{\sqrt{\Pi(1)}}\left(\left.\boldsymbol{u}_{2}\right|_{h+}-\left.u_{2}\right|_{h-}\right),
\end{aligned}
$$

where the square brackets denote the jump of the enclosed quantity across the interface, $\boldsymbol{n}$ is a unit vector normal to the interface, and $\boldsymbol{u}_{2}$ is the plane velocity vector denoting $(u, v)$. In above boundary conditions, Eq. (14a) is the liquidus relation applying at the interface, Eq. (14b) is the marginal equilibrium 
condition [16], Eq. (14c) is the continuity of normal velocity, Eqs. (14d) and (14e) are continuities of temperature and heat flux, respectively, Eq. (14f) is an enforced condition assuming the solid at the interface vanished, Eq. $(14 \mathrm{~g})$ is the continuity of normal stress including the deviatoric stress, Eq. (14h) is the Beavers-Joseph boundary condition governing the relation between the tangential velocity just above the interface $\left.u_{2}\right|_{h+}$ and that just beneath the interface $\left.u_{2}\right|_{h_{-}}$. Note that $\Lambda$ is an empirical constant ranging from 0.1 to 4 , as determined experimentally by Beavers and Joseph [17]. In a previous study [18], it was found that the value of $\Lambda$ does not significantly influence the stability characteristics of a similar flow. We thus consider $\Lambda=0.1$ in the present study. Note that as the coefficient of Eq. (14h) approaches infinity, the no-slip condition $[5,10]$ is recovered. Nevertheless, since $0.1 \leq \Lambda \leq 4, \sqrt{\Pi(1)} \approx \mathcal{O}(1)$, and $\sqrt{\mathcal{H}} \approx 10^{3}$, the coefficient is thus of order of $\mathcal{O}\left(10^{2}\right)$ $\mathcal{O}\left(10^{3}\right)$, which to some extent results in a difference between the Beavers-Joseph condition and the no-slip condition. At the mush/solid interface $z=0$ we have

$\theta=-1$,

$\left[\rho\left(\boldsymbol{u}-\boldsymbol{e}_{k}\right) \cdot \boldsymbol{n}\right]=0$,

where Eq. (15b) accounts for the conservation of mass at the interface.

The above mathematical formulation differs from that of previous studies $[5,10]$ by several respects. First, in Eq. (8) we consider the solute diffusion in the mush, which is ignored by Worster but can be important when $\epsilon$ is not small. Secondly, at the melt/mush interface, we consider the Beavers-Joseph boundary condition for the tangential velocity along the interface, rather than use the no-slip condition $[5,10]$. Thirdly, the deviatoric stress is considered in the condition of the continuity of normal stress (Eq. (14g)). These two interfacial dynamical conditions are influential to the dynamical coupling between the convection in the fluid and the mushy layers. A detailed discussion of the comparison between these two mathematical models is provided in Section 5.1.

\section{Basic state solution}

The equations and associated boundary conditions of Section 2 admit a steady solution as a function of
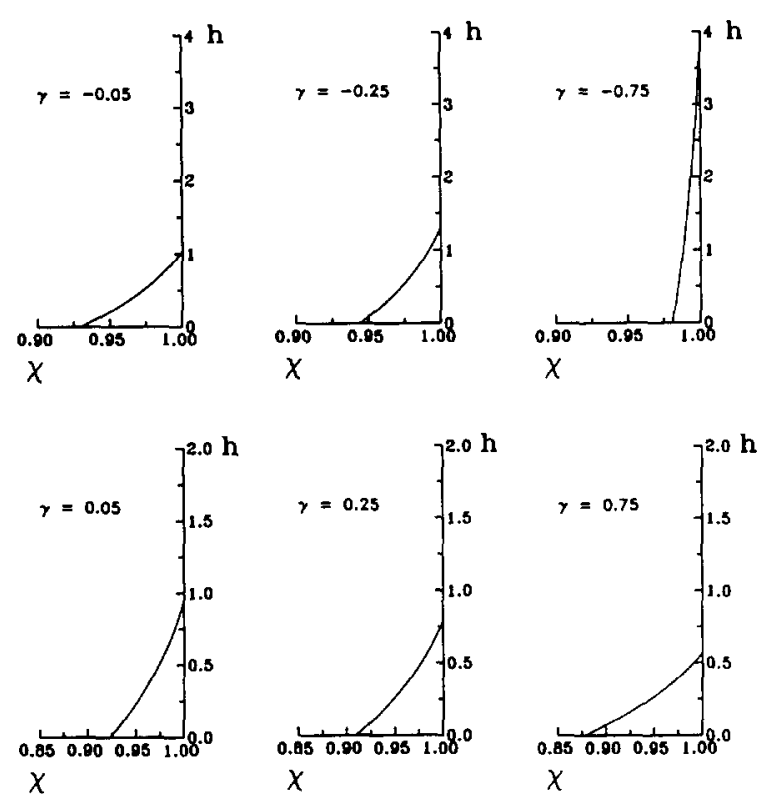

Fig. 1. Variation of basic state liquid mass fraction in the vertical direction of the mushy layer for various $\gamma$.

$z$, which is presented in the following. In the mushy layer, according to Eqs. (7) and (14b), the velocity of the interstitial fluid is

$w_{\mathrm{b}}(z)=-\gamma \chi_{\mathrm{b}}(z)$,

where the index $\mathrm{b}$ accounts for the basic state and $w$ is the vertical velocity. Since at the melt/mush interface $\chi=1$, the vertical velocity in the melt is accordingly

$w_{\mathrm{b}}=-\gamma$,

which is in accordance with the boundary condition Eq. (13c). The basic state temperature and concentration in the fluid layer can be obtained from Eqs. (2) and (3), respectively,

$\theta_{\mathrm{b}}=\theta_{\infty}+\left(\theta_{\mathrm{i}}-\theta_{\infty}\right) \mathrm{e}^{-(1+\gamma)(z-h)}$,

$\Theta_{\mathrm{b}}=\theta_{\mathrm{i}} \mathrm{e}^{-[(1+\gamma) / \epsilon](z-h)}$,

where the interfacial temperature is given by

$\theta_{\mathrm{i}}=-\frac{\epsilon}{1-\epsilon} \theta_{\infty}$.

In the mushy layer, the relation $\theta=\Theta$ applies. The liquid mass fraction of the mush $\chi_{b}$ is governed by 

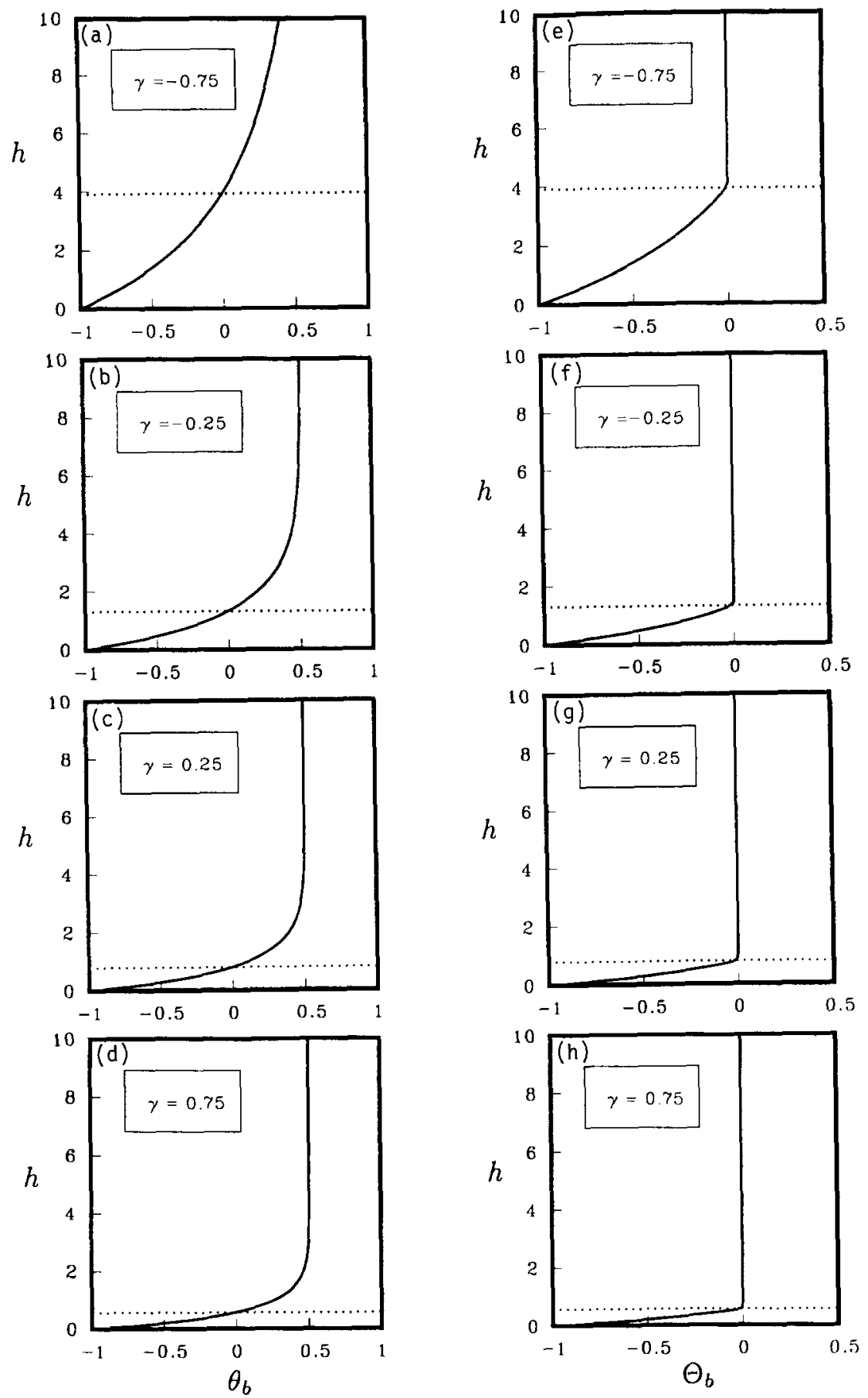

Fig. 2. Basic state temperature and concentration distribution in the vertical direction for various $\gamma$. (a)-(d): basic state temperature; (e) $-(\mathrm{h})$ : basic state concentration. 
Table 1

Physical properties and corresponding dimensionless parameters of $26 \mathrm{wt} \% \mathrm{NH}_{4} \mathrm{Cl}$ solution

\begin{tabular}{lll}
\hline$A$ & 0.65 & \\
$\mathcal{C}$ & 12.3 & $\%$ \\
$C_{\mathrm{E}}$ & 19.7 & $\mathrm{~J} / \mathrm{kg}^{\circ} \mathrm{C}$ \\
$c$ & 3249 & \\
$\mathcal{F}$ & 3.20 & \\
$\mathcal{H}$ & $3.5 \times 10^{6}$ & $\mathrm{~J} / \mathrm{kg}$ \\
$\mathcal{L}$ & $3.14 \times 10^{5}$ & ${ }^{\circ} \mathrm{C}$ \\
$T_{\mathrm{E}}$ & -15.4 & ${ }^{\circ} \mathrm{C}^{-1}$ \\
$\alpha^{*}$ & $3.832 \times 10^{-4}$ & $\%{ }^{-1}$ \\
$\beta^{*}$ & $2.82 \times 10^{-3}$ & ${ }^{\circ} \mathrm{C} / \%$ \\
$\Gamma$ & 4.75 & \\
$\gamma$ & -0.31 & \\
$\epsilon$ & 0.013 & \\
$\theta_{\infty}$ & 0.5 & $\mathrm{~cm}^{2} / \mathrm{s}$ \\
$\kappa_{\mathrm{tl}}$ & $1.336 \times 10^{-3}$ & \\
$\sigma$ & 10. & $\mathrm{C}^{-3}$ \\
\hline
\end{tabular}

$\chi_{\mathrm{b}}=\frac{-\mathcal{C}}{(1+\gamma)\left(\theta_{\mathrm{b}}-\mathcal{C}\right)+\epsilon \theta_{\mathrm{b}}^{\prime}+\gamma \mathcal{C}}$,

which can be deduced from Eq. (18), and the basic state temperature is governed by

$\theta_{\mathrm{b}}^{\prime \prime}+\left(1+\gamma_{\chi_{\mathrm{b}}}\right) \theta_{\mathrm{b}}^{\prime}-\mathcal{F}\left[\rho_{\mathrm{b}}\left(1-\chi_{\mathrm{b}}\right)\right]^{\prime}=0$,

which is derived from Eq. (9). Eqs. (21) and (22) and associated boundary conditions are solved with a shooting technique, in which the interface position $h$ is also solved implicitly.

In this study, we focus on the volume change effect on the convective stability of a $26 \mathrm{wt} \% \mathrm{NH}_{4} \mathrm{Cl}$ solution, whose physical properties are listed in Table 1. Using these data, the basic state solutions for various $\gamma$ are obtained as follows. Fig. 1 shows the distribution of $\chi$ in the vertical direction and the corresponding mushy layer height $h$. For $\gamma<0$ (Figs. 1a-c), an upward flow is induced, both $\chi$ and $h$ increases as $\gamma$ decreases. This is due to the fact that the upward flow convectively carries cold and less concentrated fluid from below, enhancing the mushy-layer growth while decreasing the solid mass fraction in the mush. On the other hand, as $\gamma>0$ (Figs. 1d-f), a downward flow is induced and both $\chi$ and $h$ decrease with increasing $\gamma$. This is because the downward flow brings warmer and more concentrated fluid from the bulk fluid into the mush, suppressing the mushy-layer growth while increasing the solid mass fraction of the mush. Fig. 2 illustrates the basic state temperature and concentra- tion for various $\gamma$. It is seen that increasing $\gamma$ enhances both the temperature and concentration gradients in the mush and the temperature gradient in the melt as well. The increase of the two gradients in the mush is due to the decrease of the mushy-layer depth. The increase of the temperature gradient in the melt is also due to the reduction of the thermal boundary-layer thickness.

\section{Small disturbance equations and method of solution}

We linearize the equations of Section 2 by introducing small disturbances to the basic state quantities of Section 3 and substituting their combination into the original equations. After obtaining the linearized equations by neglecting higher order terms and appling the normal modes proportional to $\mathrm{e}^{\omega t+\mathrm{i} \alpha x}$, the small disturbance equations are as follows. In the fluid layer, we have

$\left(D^{2}-\alpha^{2}\right) W=\Omega$,

$$
\begin{aligned}
& {\left[D^{2}+\frac{(1+\gamma) D-\omega}{\sigma}-\alpha^{2}\right] \Omega} \\
& \quad=\alpha^{2} \mathcal{H} R_{\mathrm{m}}[A \theta-(1+A) \Theta], \\
& {\left[D^{2}+(1+\gamma) D-\omega-\alpha^{2}\right] \theta=\theta_{\mathrm{b}}^{\prime} W} \\
& {\left[\epsilon D^{2}+(1+\gamma) D-\omega-\epsilon \alpha^{2}\right] \Theta=\Theta_{\mathrm{b}}^{\prime} W .}
\end{aligned}
$$

In these equations, the notations $W, \theta, \Theta$ now represent small disturbance quantities. In addition, both ' and $D$ represent the vertical derivative $\mathrm{d} / \mathrm{d} z, \alpha$ is the horizontal wave number, $\omega$ is the normal mode frequency, $\Omega$ is the disturbance vorticity, $\mathcal{H}=H^{2} / \Pi$, $A=\Gamma \alpha^{*} / \beta$ is the buoyancy ratio between the buoyancy due to concentration to that due to temperature, $R_{\mathrm{T}}=A \mathcal{H} R_{\mathrm{m}}$, and $R_{\mathrm{C}}=(1+A) \mathcal{H} R_{\mathrm{m}}$.

Similarly, in the mushy layer we have

$$
\begin{gathered}
\epsilon \chi_{\mathrm{b}}\left(1+\gamma \chi_{\mathrm{b}}\right) D^{2} \theta+\left[\epsilon \theta_{\mathrm{b}}^{\prime}+(1+\gamma)\left(\theta_{\mathrm{b}}-\mathcal{C}\right)\right] D \chi \\
=\left[-\epsilon \chi_{\mathrm{b}}^{\prime}-(1+\gamma)\left(1+\gamma \chi_{\mathrm{b}}\right) \chi_{\mathrm{b}}\right] D \theta \\
+\left[\left(\epsilon \alpha^{2}+\omega\right)\left(1+\gamma \chi_{\mathrm{b}}\right) \chi_{\mathrm{b}}-(1+\gamma) \chi_{\mathrm{b}}^{\prime}\right] \theta
\end{gathered}
$$


$+\left[-\epsilon\left(\theta_{\mathrm{b}}^{\prime \prime}-2 \gamma \chi_{\mathrm{b}}^{\prime} \theta_{\mathrm{b}}^{\prime}+\frac{2 \gamma^{2} \chi_{\mathrm{b}} \chi_{\mathrm{b}}^{\prime} \theta_{\mathrm{b}}^{\prime}}{1+\gamma \chi_{\mathrm{b}}}\right)\right.$

$+\left(\theta_{\mathrm{b}}-\mathcal{C}\right)(1+\gamma)\left(\omega+\frac{2 \gamma \chi_{\mathrm{b}}^{\prime}}{1+\gamma \chi_{\mathrm{b}}}\right)$

$\left.+\left(\gamma^{2} \chi_{\mathrm{b}}-1\right) \theta_{\mathrm{b}}^{\prime}\right] \chi+\left(1+\gamma_{\chi_{\mathrm{b}}}\right) \theta_{\mathrm{b}}^{\prime} W$,

$$
\begin{aligned}
(1+ & \left.\gamma \chi_{\mathrm{b}}\right)^{2} D^{2} \theta+\mathcal{F}(1+\gamma)^{2} D \chi \\
= & -\left(1+\gamma \chi_{\mathrm{b}}\right)^{3} D \theta+\left(1+\gamma \chi_{\mathrm{b}}\right)^{2}\left(\alpha^{2}+\omega\right) \theta \\
& +\mathcal{F}(1+\gamma)^{2}\left(\omega+\frac{2 \gamma \chi_{\mathrm{b}}^{\prime}}{1+\gamma \chi_{\mathrm{b}}}\right) \chi \\
& +\left(1+\gamma \chi_{\mathrm{b}}\right)^{2} \theta_{\mathrm{b}}^{\prime} W,
\end{aligned}
$$

$$
\begin{aligned}
D^{2} W & +\gamma D^{2} \chi \\
= & \left(\frac{\gamma}{1+\gamma \chi_{\mathrm{b}}}+\frac{\Pi^{\prime}}{\Pi}\right) \chi_{\mathrm{b}}^{\prime} D W \\
& +\gamma\left(\frac{\omega+\gamma \chi_{\mathrm{b}}^{\prime}}{1+\gamma_{\mathrm{b}}}+\frac{\Pi^{\prime} \chi_{\mathrm{b}}^{\prime}}{\Pi}\right) D_{\chi} \\
& -\left[\frac{\gamma}{1+\gamma \chi_{\mathrm{b}}}\left(\frac{\Pi^{\prime} \chi_{\mathrm{b}}^{\prime 2}}{\Pi}-\chi_{\mathrm{b}}^{\prime \prime}\right)\right. \\
& \left.+\left(\frac{\gamma \chi_{\mathrm{b}}^{\prime}}{1+\gamma \chi_{\mathrm{b}}}\right)^{2}-\alpha^{2}\right] W \\
& +\alpha^{2} R_{\mathrm{m}} \Pi\left(\chi_{\mathrm{b}}\right) \theta-\frac{\gamma^{2}}{\left(1+\gamma \chi_{\mathrm{b}}\right)^{2}} \\
& \times\left[\left(\omega+\gamma^{2} \chi_{\mathrm{b}}^{\prime}\right) \chi_{\mathrm{b}}^{\prime}-\left(1+\gamma \chi_{\mathrm{b}}\right) \chi_{\mathrm{b}}^{\prime \prime}\right. \\
& \left.+\frac{\Pi^{\prime}}{\Pi} \chi_{\mathrm{b}}^{\prime}\left(\omega+\gamma \chi_{\mathrm{b}}^{\prime}\right)\left(1+\gamma \chi_{\mathrm{b}}\right)\right] \chi .
\end{aligned}
$$

In above equations, the disturbance density has been replaced by the disturbance mass fraction by the relation

$\rho(z)=-\frac{\gamma(1+\gamma)}{\left(1+\gamma \chi_{\mathrm{b}}(z)\right)^{2}} \chi(z)$,

where now $\rho$ and $\chi$ are the small disturbance quantities. This relation is obtained from the Taylor expansion of Eq. (12) with respect to the basic state.

The boundary conditions at $z \rightarrow \infty$ are

$$
\begin{aligned}
& \theta=0, \\
& \Theta=0,
\end{aligned}
$$

$W=0$

$D W=0$,

at $z=h$ are

$\theta=\Theta$

$D \Theta-D \theta=(1+\gamma)\left(\frac{1-\epsilon}{\epsilon}\right) \theta_{\mathrm{b}}^{\prime} \eta$,

$[W]=0$,

$[\theta]=0$,

$[D \theta]=-(1+\gamma) \frac{\mathcal{F} \theta_{\mathrm{b}}^{\prime}}{\mathcal{C}-\theta_{\mathrm{i}}} \eta$,

$\chi=-\chi_{\mathrm{b}}^{\prime} \eta$

and at $z=0$ are

$\theta=0$,

$W=-\gamma \chi$,

where $\eta$ is the small perturbation of the interface position. At the melt/mush interface the continuity of normal stress is

$$
\begin{aligned}
{[D W} & \left.-\frac{\gamma \chi_{\mathrm{b}}^{\prime}}{1+\gamma} W+\gamma D \chi-\frac{\gamma\left(\omega+\gamma \chi_{\mathrm{b}}^{\prime}\right)}{1+\gamma} \chi\right]_{h-} \\
= & -\frac{\Pi(1)}{\mathcal{H}}\left[D \Omega+\frac{1+\gamma}{\sigma}\left(\Omega+\alpha^{2} W\right)\right. \\
& \left.-\frac{\omega}{\sigma} D W-2 \alpha^{2} D W\right]_{h+},
\end{aligned}
$$

in which the deviatoric stress is considered. The Beavers-Joseph boundary condition is given as

$$
\begin{aligned}
\left.D^{2} W\right|_{h+}= & \Lambda \sqrt{\mathcal{H}}\left[\left.D W\right|_{h+}+\frac{\gamma^{2} \chi_{\mathrm{b}}^{\prime}}{1+\gamma} \chi-\gamma D \chi\right. \\
& \left.+\frac{\gamma \chi_{\mathrm{b}}^{\prime}}{1+\gamma} W-\left.D W\right|_{h-}+\frac{\gamma}{(1+\gamma)} \omega \chi\right]
\end{aligned}
$$

The eigenvalue problem is composed of Eqs. (23)(29) and Eqs. (31)-(35) and 14 boundary conditions, in which Eq. (32b) is used to solve the disturbance interface position $\eta$. We solve this complex eigenvalue problem with a shooting method. The integration is implemented in a truncated domain [19], in which the boundary conditions at $z \rightarrow \infty$ are imposed at a finite distance $z=20$, which is sufficiently large so that the results are independent of the value 
(a)

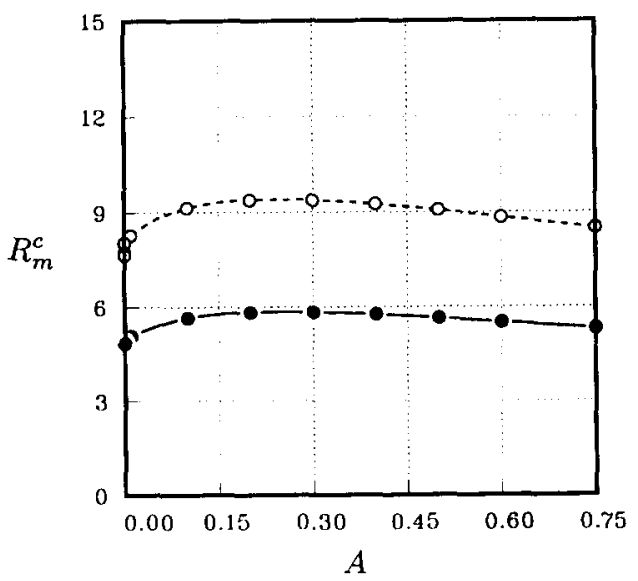

(b)

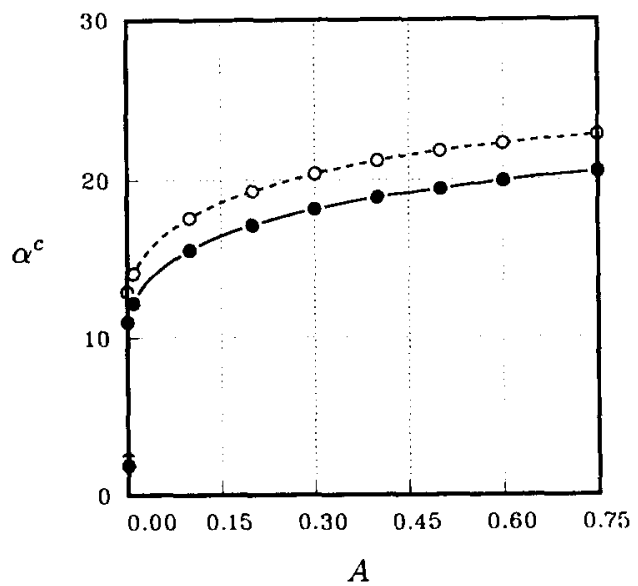

Fig. 3. Comparison between the results obtained from Worster's (1992) model (dash) and the present model (solid) for an alloy of $\mathcal{F}=\mathcal{C}=\theta_{\infty}=1, \sigma=10, \epsilon=0.025, \mathcal{H}=10^{5}$, and $\Pi=1$. The curves show the variations of $R_{\mathfrak{m}}^{c}$ and $\alpha^{\mathrm{c}}$ with $A$, the buoyancy ratio.

of $z$. The two-layer configuration is converted into a single layer by the superposition method developed by Keller [20], in which an orthonormalization process is applied to avoid the loss of linear independence of integration. This method was later improved and called parallel shooting by Davey [21] who developed the automatic orthonormalization process. An iteration procedure developed by Powell [22] is employed to seek the convergence of the eigenvalues $R_{\mathrm{m}}$ and $\omega_{\mathrm{i}}$.

The present eigenvalue problem has some inherent difficulties. For example, the physical domain consists of two different layers, the equations of different layers have different characteristic scales, and so on. The difficulties are especially prevalent in the high $\alpha$ domain and on the Hopf bifurcation branch as well. To obtain a point on the monotonic branch curve for $\alpha>$ 30 , for instance, about 250 CPU seconds of CRAY YMP/EL is essential. For a point on the Hopf bifurcation branch, more than $600 \mathrm{CPU}$ seconds are required. More severely, the initial guess of $\omega_{\mathrm{i}}$ needs to be within $20 \%$ of the final solution.

\section{Results and discussion}

\subsection{Assessment of mathematical model}

The present mathematical formulation can be employed to investigate the problem of Worster [5] when $\gamma=0$ (so that the liquid mass fraction is equal to the liquid volume fraction), the solute diffusion in Eq. (8) is neglected, the Beavers-Joseph condition Eq. (14h) is replaced by the no-slip condition, and the deviatoric term in Eq. (14g) is dropped. The computer code written according to this reduced model is employed to run the case considered in Worster, i.e., $\mathcal{F}=\mathcal{C}=$ $\theta_{\infty}=1, \sigma=10, \epsilon=0.025, \mathcal{H}=10^{5}, A=0$, and a uniform permeability $\Pi(\chi)=1$. The neutral curve we obtain, a monotonic one in which the mushy-layer mode is more unstable than the boundary-layer mode, is exactly the same as that of Fig. 3 of Worster. We then compute the critical Rayleigh number $R_{\mathrm{m}}^{\mathrm{c}}$ and critical wave number $\alpha^{\mathfrak{c}}$ for non-zero $A$ and the results are illustrated with the dashed curves in Fig. 3. For the physical significance of $A$, the reader is referred to Chen, Lu and Yang [10], in which a detailed discussion is provided.

We then consider the present mathematical formulation for the same case and the results are shown in the same figure with solid curve. Note that for both curves of Fig. 3 the values of $R_{\mathrm{m}}^{\mathrm{c}}$ and $\alpha^{\mathrm{c}}$ are corresponding to the boundary-layer mode convection except the point of $A=0$ where the mushy-layer mode convection prevails. Results show that the $R_{\mathrm{m}}^{\mathrm{c}}$ of present study is in general $30 \%$ lower than that of Worster [5]. Since the value of $\epsilon$ of the present case is small, the influence of the solute diffusion in the mush is negligible. 

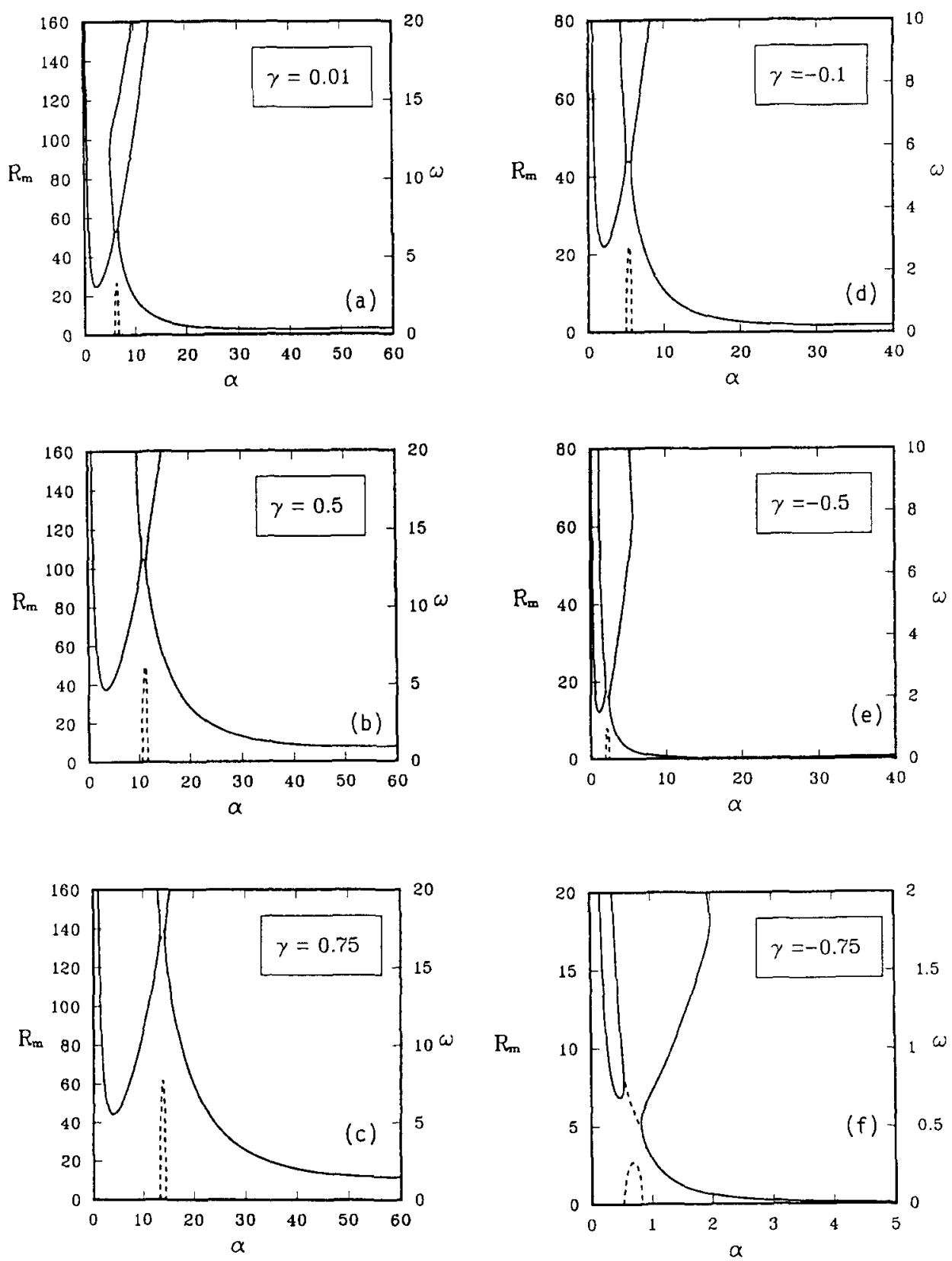

Fig. 4. The neutral curve topology for various $\gamma$; solid: monotonic mode; dot: oscillatory mode; dash: oscillation frequency. Note that the curve of oscillatory mode lies between the two curves of monotonic mode for all the sub-figures. 
The deviatoric stress in Eq. (14g) and Beavers-Joseph condition of Eq. (14h), nevertheless, are responsible for the difference shown in Fig. 3. Because, firstly, the normal gradient of the vertical disturbance velocity at the interface is not at all small if the boundarylayer mode convection predominates, which is the case when $A>0$ (see Fig. 1 of Chen, Lu and Yang [10]); secondly, the Beavers-Joseph condition allows a slip of tangential velocity along the interface, which compared to the no-slip condition $[5,10]$ is less restrictive to the flow and thus leads to a more unstable situation. In addition to the stability criteria, these two boundary conditions can also be influential on the dynamic coupling between the convection in the fluid and the mushy layers, which is especially important to the oscillatory mode on the Hopf bifurcation branch since in this mode the convection cells in the two layers are viscously coupled through the interface. This shall be discussed in the next section.

\subsection{Stability of $\mathrm{NH}_{4} \mathrm{Cl}$ solution cooling from below}

In this section, a $26 \mathrm{wt} \% \mathrm{NH}_{4} \mathrm{Cl}$ solution is considered, whose physical properties are listed in $\mathrm{Ta}-$ ble 1 . Since no experimental data are directly available to identify the permeability function $\Pi(\chi)$ of the mush of $\mathrm{NH}_{4} \mathrm{Cl}$ solution, we therefore consider for simplicity a non-uniform permeability $\Pi=\chi^{3}$ to account for the mushy-layer morphology; this is similar to Fowler [13] who assumed $\Pi=\chi^{2}$. Note that the use of present permeability model may well simulate the dendritic matrix of the mushy zone of experiment, especially on the melt/mush interface on which $x=1$. The effect on the stability due to $\gamma$ ranging from 0.75 to -0.75 is considered and $\gamma \approx-0.3$ for $26 \mathrm{wt} \% \mathrm{NH}_{4} \mathrm{Cl}$ solution [9]. In Fig. 4, several selected neutral curves of various $\gamma$ are illustrated. It is seen, by and large, that the neutral curve consists of two separated monotonic branches and a Hopf bifurcation branch sitting in between, the boundary-layer mode (high $\alpha$ mode) is invariably more unstable than the mushy-layer mode (low $\alpha$ mode), and the oscillatory mode never predominates. When $\gamma$ is less than -0.75 (Fig. 4f) the mushy-layer mode virtually vanishes, leaving the boundary-layer mode predominates. We summarize both $\alpha^{\mathrm{c}}$ and $R_{\mathrm{m}}^{\mathrm{c}}$ of various $\gamma$ in Fig. 5, showing that the basic state becomes more stable as $\gamma$ increases, and that the critical wave num-
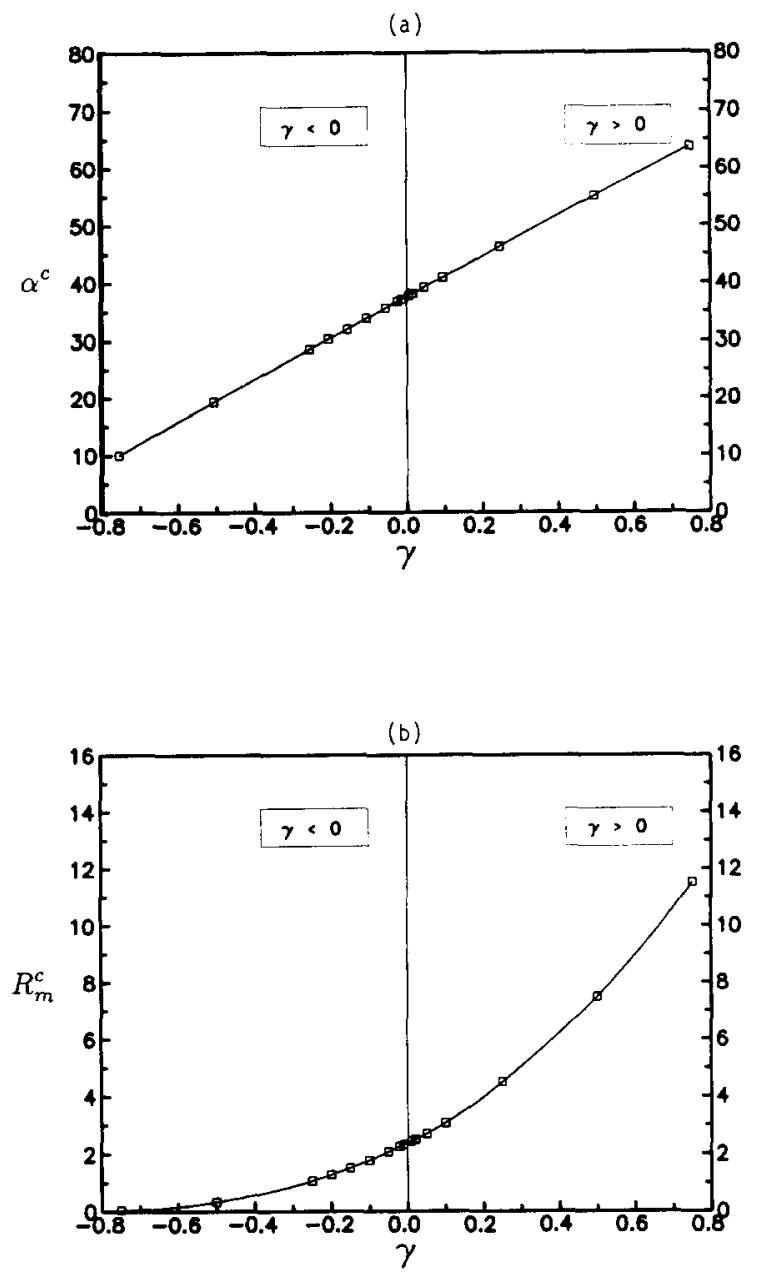

Fig. 5. The variation of $\alpha^{\mathfrak{c}}$ and $R_{\mathrm{m}}^{c}$ for various $\gamma$.

ber increases linearly with $\gamma$ as well. Fig. 6 shows a typical onset streamline pattern of the two instability modes of $\gamma=-0.1$ (see Fig. $4 \mathrm{~d}$ for the corresponding neutral curve). In this figure, the most unstable boundary-layer mode is demonstrated with solid lines and the mushy-layer mode is shown with shadow. The horizontal wave length is normalized with respect to the mushy-layer thickness, on which all dimension are scaled. One can see that the boundary-layer mode convection is largely confined to the small boundary-layer above the melt/mush interface while the mushy-layer mode circulates between the fluid and mushy layers although largely confined to the latter. In the present case, the basic flow induced by the volume change does not significantly influence the onset flow. 


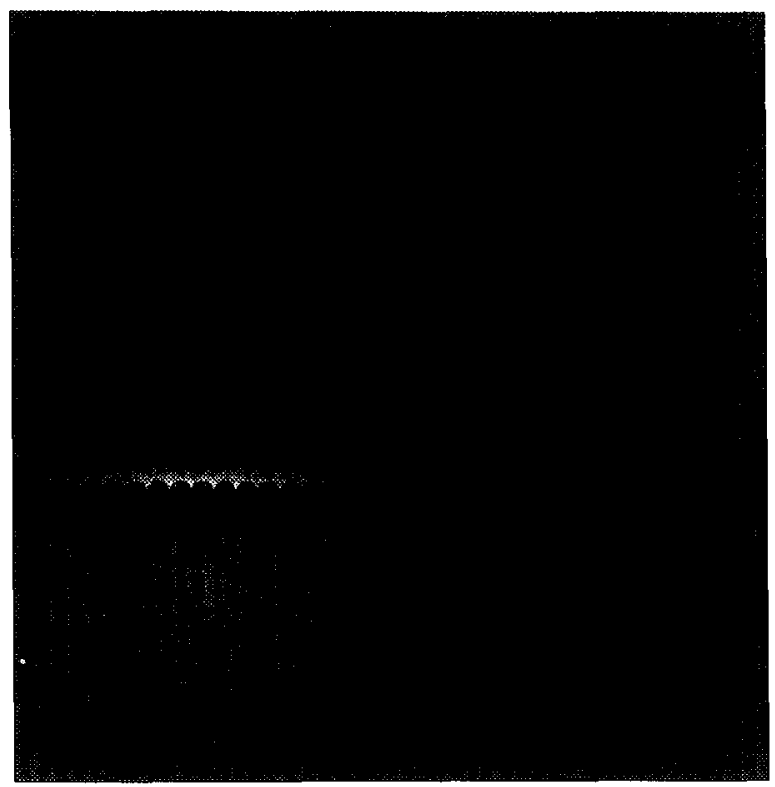

Fig. 6. The critical streamline patterns of the boundary-layer mode (solid) and the mushy-layer mode (shadow) of $\gamma=-0.1$ The cells at the left and the right are circulating in opposite sense.

The physical explanation to the above results are proposed in the following. For $\gamma>0$, a downward flow is induced. The boundary-layer mode becomes more stable as $\gamma$ increases since both the thermal and solutal boundary layers are reduced in thickness, leading to a more stable situation. The mushy-layer mode also becomes more stable as $\gamma$ increases. This is due to the fact that the mushy-layer mode is largely confined to the mushy layer in which the porosity decreases as $\gamma$ increases, resulting in a more stable situation. The critical wave number $\alpha^{\mathrm{c}}$, inversely proportional to the critical wavelength, of both modes increases with $\gamma$ because both the boundary layer and the mushy layer, to which the instability modes are largely confined, are reduced in thickness as $\gamma$ increases (see Figs. 1 and 2). The linear relation between $\alpha^{\mathrm{c}}$ and $\gamma$ is due to the fact that the compositional boundary-layer thickness decreases approximately linearly with increasing $\gamma$, which we determine from the numerical results. For $\gamma<0$, an upward flow is induced. For the boundarylayer mode, as $\gamma$ decreases, a stronger upward flow increases the thermal boundary layer thickness, leading to a decrease of the stabilizing thermal gradient and thus a more unstable state. The mushy-layer mode also becomes more unstable as $\gamma$ decreases since the

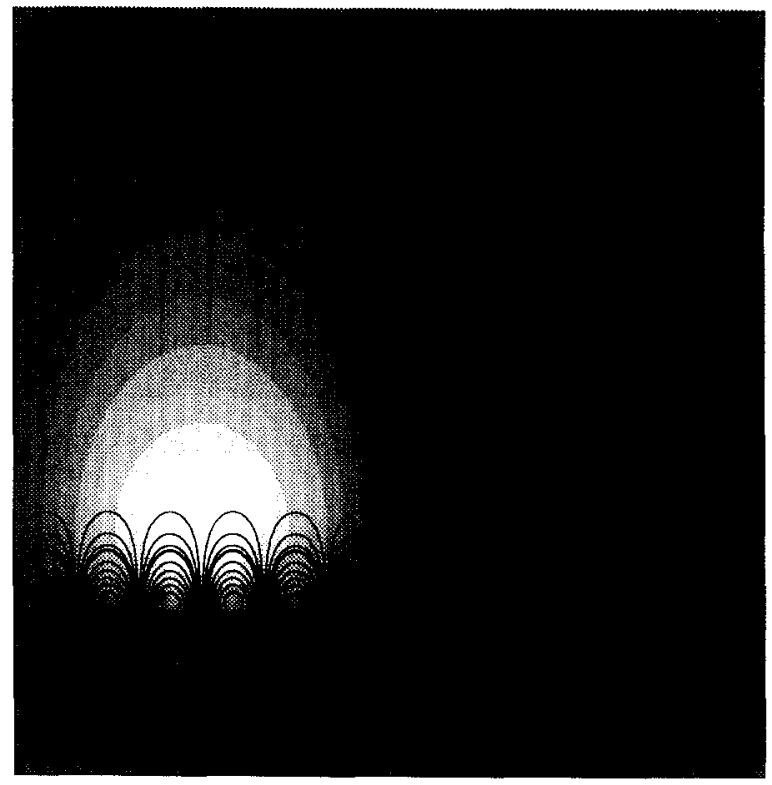

Fig. 7. The critical streamline patterns of the boundary-layer mode (solid) and the mush-layer mode (shadow) of $\gamma=-0.75$. The cells at the left and the right are circulating in opposite sense.

porosity increases.

To gain more physical insights into the $\gamma$ effect, we present another onset streamline pattern of $\gamma=$ -0.75 in Fig. 7. Note that in reality the mushy-layer thickness of Fig. 7 shall be larger than that of Fig. 6, which is nevertheless not reflected in these two figures. This is due to the difficulty that if the two figures are scaled with, for instance, the mushy-layer thickness of $\gamma=-0.1$, the size of Fig. 7 shall be much larger than the present one; and vice versa when scaled with (a)

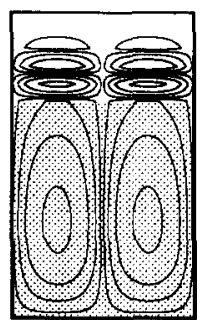

(b)

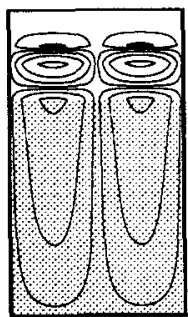

(c)

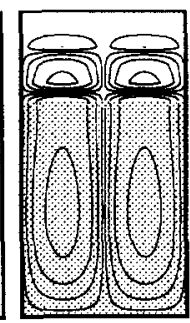

Fig. 8. The onset streamline patterns of oscillatory mode of $\gamma=0.75$. (a) $\alpha=13.24$; (b) $\alpha=13.84$; (c) $\alpha=14.42$. Note that the oscillating frequency is largest in case (b) since the convection in the mush is the weakest in the three cases considered. 
(a)

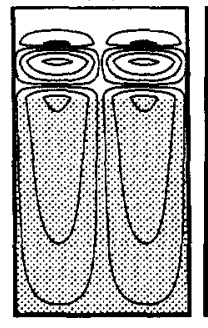

(b)
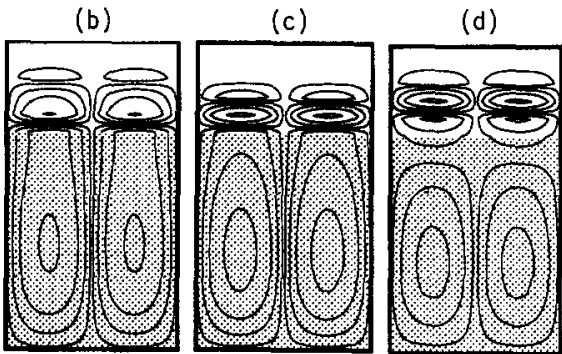

Fig. 9. The onset streamline patterns of oscillatory mode of $\gamma=0.75$ and $\alpha=13.84$. (a) $t=0$; (b) $t=\pi / 4$; (c) $t=\pi / 2$; (d) $t=3 \pi / 4$

\section{Conclusion}

We have proposed a different mathematical model to investigate the volume change effect on the saltfinger instability of directionally solidifying $\mathrm{NH}_{4} \mathrm{Cl}$ solution. It is found that the induced basic flow changes both the morphology and the depth of the mush, which in turn influences the stability characteristics of the onset of salt-finger convection. More precisely, for $\gamma>0$, a downward flow is induced; both the boundary-layer and the mushy-layer modes become more stable as $\gamma$ increases; for $\gamma<0$, an upward flow is induced, both modes become less stable as $\gamma$ decreases. For $\gamma<-0.75$, the mushy-layer mode virtually vanishes, implying that the plume convection, a direct result of the subcritical mushy-layer mode convection, may be more difficult to occur.

\section{Acknowledgement}

The financial support for this work from Nation Science Council through Grant No NSC 83-0401-E002-005 is gratefully acknowledged.

\section{References}

[1] A.K. Sample and A. Hellawell, Met. Trans. 15 A (1984) 2163.

[2] S. Tait and C. Jaupart, Nature 338 (1989) 571.

[3] C.F. Chen and F. Chen, J. Fluid Mech. (1991) 227, 567.

[4] D.G. Neilson and F.P. Incropera, Intern. J. Heat Mass Transfer 34 (1991) 1717.

[5] M.G. Worster, J. Fluid Mech. 237 (1992) 649.

[6] G. Amberg and G.M. Homsy, J. Fluid Mech. 252 (1993) 79.

[7] C.S. Magirl and F.P. Incropera, J. Heat Transfer ASME Trans. C 115 (1993) 1036.

[8] M.H. McCay, T.D. McCay and J.A. Hopkins, Met. Trans. B 24B (1993) 669.

[9] A. Hellawell, J.R. Sarazin and R.S. Steube, Phil. Trans. Royal Soc. (London) A 345 (1993) 507.

[10] F. Chen, J.W. Lu and T.L. Yang, J. Fluid Mech. 276 (1994) 163.

[11] P. Nandapurkar, D.R. Poirier, J.C. Heinrich and S. Felicelli, Met. Trans. B 20B (1989) 711.

[12] S.H. Davis, J. Fluid Mech. 212 (1990) 241.

[13] A.C. Fowler, IMA J. Appl. Math. 35 (1985) 159.

[14] A.O.P. Chiareli and M.G. Worster, J. Crystal Growth 125 (1992) 487.

[15] G.B. McFadden and S.R. Coriell, Phys. Fluids 30 (1987) 659.

[16] M.G. Worster, J. Fluid Mech. 167 (1986) 481. 
[17] G.S. Beavers and D.D. Joseph, J. Fluid Mech. 30 (1967) 197.

[18] F. Chen, J. Appl. Phys. 71 (1992) 5222.

[19] S.R. Coriell, M.R. Cordes, W.J. Boettinger and R.F. Sekerka, J. Crystal Growth 49 (1980) 13.

[20] H.B. Keller, Regional Conference Series in Applied Math., SIAM, Philadelphia, PA (1976).
[21] A. Davey, J. Comp. Phys. 51 (1983) 343.

[22] M.J.D. Powell, Numerical Methods for Nonlinear Algebraic Equations, Ed. P.H. Rabinowitz (Gordon and Breach, New York, 1970).

[23] S. Rasenat, F.H. Busse and I. Rehberg, J. Fluid Mech. 199 (1989) 519. 